Editorial

\title{
Mechanics, Fatigue, and Fracture of Structural Joints
}

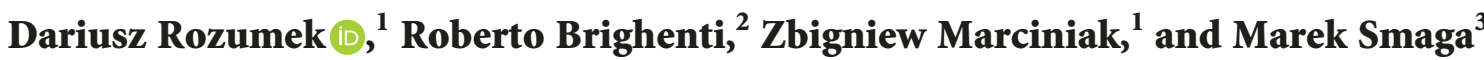 \\ ${ }^{1}$ Opole University of Technology, Opole, Poland \\ ${ }^{2}$ University of Parma, Parma, Italy \\ ${ }^{3}$ Technische Universität Kaiserslautern, Kaiserslautern, Germany \\ Correspondence should be addressed to Dariusz Rozumek; d.rozumek@po.opole.pl
}

Received 16 April 2019; Accepted 16 April 2019; Published 5 May 2019

Copyright ( 2019 Dariusz Rozumek et al. This is an open access article distributed under the Creative Commons Attribution License, which permits unrestricted use, distribution, and reproduction in any medium, provided the original work is properly cited.

For centuries, engineering structures have been made for people's life everyday needs, such as buildings, transportation, construction equipment, infrastructures, and plants. To achieve the desired functionality, usually simple individual members are connected with each other to get the final complex desired structure. Depending on the design requirements, the structural elements can be connected in a nonpermanent or permanent manner. To ensure an adequate safety level and optimal structural joints durability, construction node tests and simulations are required to determine the effect of the various factors influencing the durability and strength of the joints. The types of different connections that have been developed and proposed to date is very huge; joints represent a crucial part of complex structures, and the consequence of their collapse can be the loss of human lives and economic resources.

Studies and research outcomes carried out in this field are necessary to guide the development of new and advanced standards for a better design and improved safety level of structural joints, leading to beneficial effects in all the technologies where connection of structural elements is required.

This special issue aims to gather the most recent research advancements in the field of structural joints, with particular emphasis on their safety and reliability. In the analysis of joints, the study of initiation and fatigue crack growth in metallic permanent structural connections is of primary interest, but studies related to any other engineering material employed in bearing joint applications are also well fitted with the aim and scope of this special issue.

Over the last year, we have received and analyzed 39 submitted research works by outstanding international scientists. The papers submitted by the authors have been subjected to the rigorous journal peer-review process. During the intensive process of reviews and in-depth discussions, 8 articles were accepted for the publication, while other documents were rejected mainly due to the discrepancy to the topic of the special issue. Accepted papers, after passing the positive review process as well as the editorial processes, will be published in this special issue of Advances in Materials Science and Engineering entirely devoted to the mechanics of structural joints.

The topics considered in the accepted papers include several aspects influencing the performance of structural joints such as the effects of factors affecting the fatigue life and crack initiation in welded joints, the influence of geometric effects (notches, fillets, etc.) on the fatigue crack growth, mechanical properties optimization, the effect of all the various factors (physical, chemical, and environmental) on the lifetime of welds, mechanics of joints made of nonmetallic materials such as concrete and polymers, whose investigation has been performed by adopting theoretical, numerical, and experimental approaches.

\section{Conflicts of Interest}

The Guest Editorial team of this special issue declares that there are no conflicts of interest or private agreements with companies regarding our work for this special issue. They have no financial relationships through employment, consultancies, and either stock ownership or honoraria, with the industry. 


\section{Acknowledgments}

We thank the reviewers who assisted us in the review process providing useful comments and proposing constructive improvements to the articles. We are grateful to all authors for their excellent contributions to this special issue.

Dariusz Rozumek

Roberto Brighenti Zbigniew Marciniak Marek Smaga 


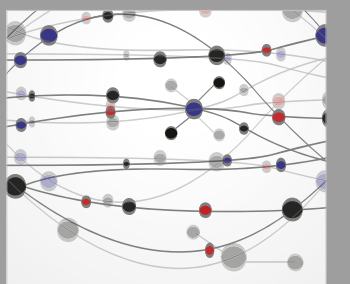

The Scientific World Journal
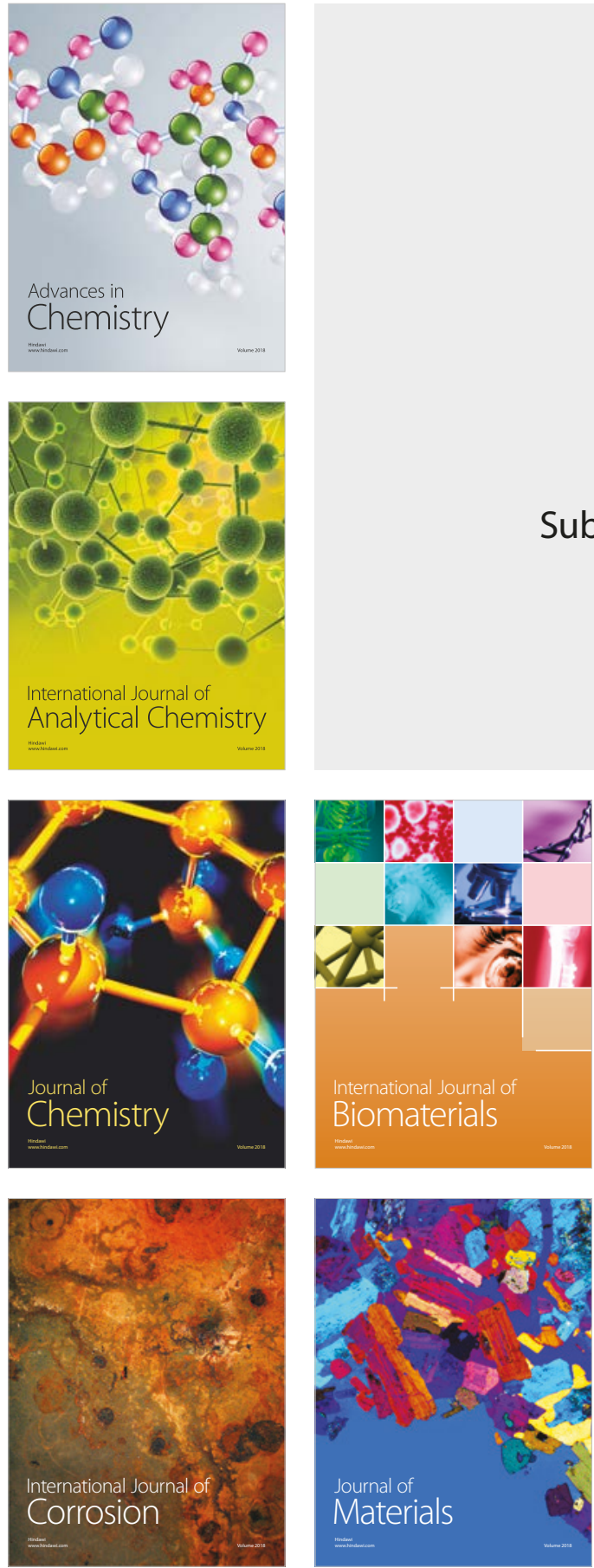

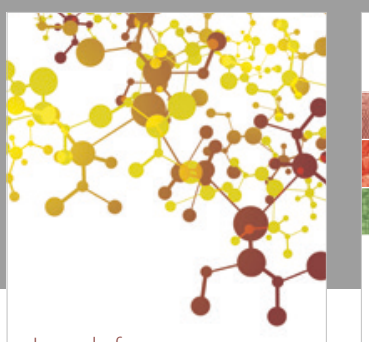

Journal of

Applied Chemistry
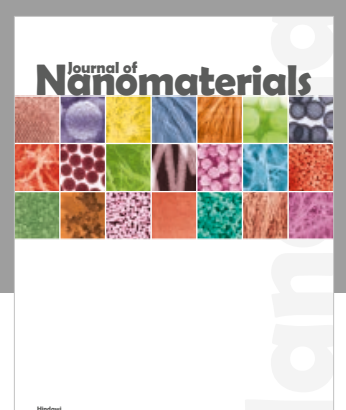

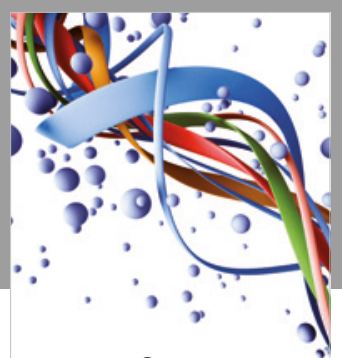

Scientifica

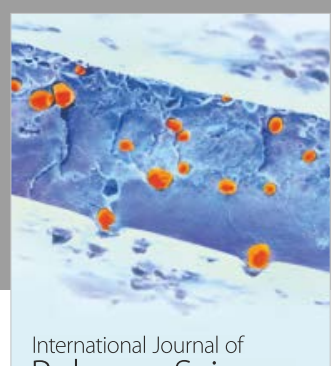

Polymer Science

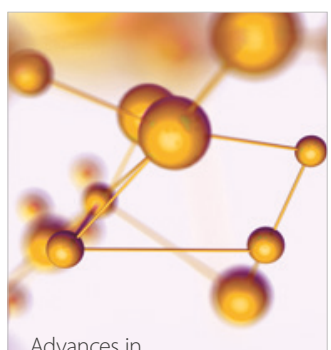

Physical Chemistry
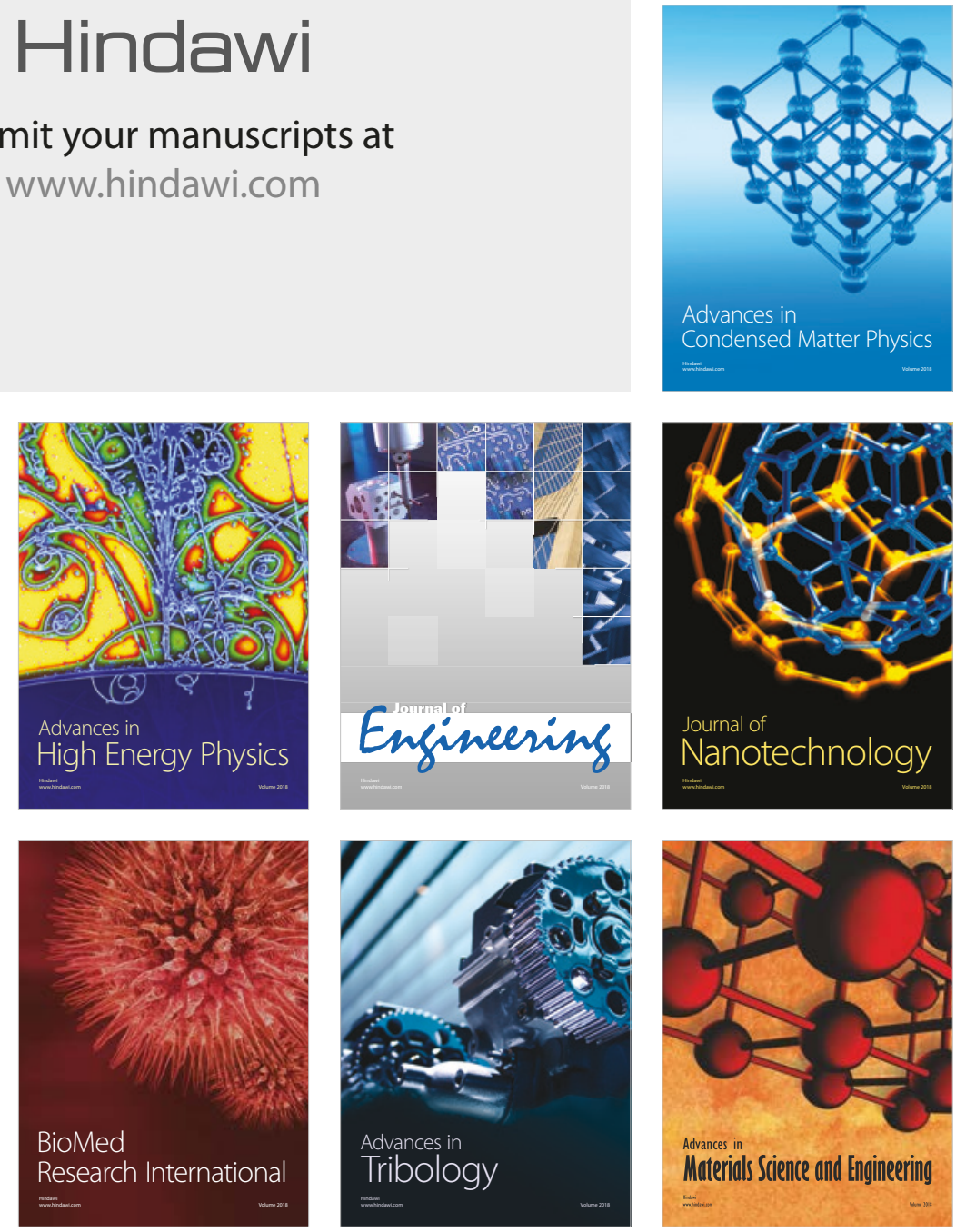